\title{
Combustion-generated nanoparticles produced in a benzene flame: A multiscale approach
}

\author{
Angela Violi ${ }^{\mathrm{a})}$ and Arun Venkatnathan ${ }^{\mathrm{b})}$ \\ Department of Mechanical Engineering, University of Michigan, Ann Arbor, Michigan 48109
}

(Received 16 May 2006; accepted 26 June 2006; published online 1 August 2006)

\begin{abstract}
This paper details the multiscale methodology developed to analyze the formation of nanoparticles in a manner that makes it possible to follow the evolution of the structures in a chemically specific way. The atomistic model for particle inception code that combines the strengths of kinetic Monte Carlo and molecular dynamics is used to study the chemical and physical properties of nanoparticles generated in a premixed fuel-rich benzene flame, providing atomistic scale structures (bonds, bond angles, dihedral angles) as soot precursors evolve into a three-dimensional structure. Morphology, density, porosity, and other physical properties are computed. Two heights corresponding to two different times in the benzene flame, experimentally studied by Bittner and Howard [Proc. Combust. Inst. 18, 1105 (1981)], were chosen to examine the influence of different environments on structural properties of the particles formed. (C) 2006 American Institute of Physics.
\end{abstract}

[DOI: $10.1063 / 1.2234481]$

\section{INTRODUCTION}

Soot formation remains one of the most challenging subjects in combustion science. Carbon emissions from the US alone amount to nearly $1.6 \mathrm{Mt}$ (megaton)/yr that pollutes the air and contributes to global warming. ${ }^{1,2}$ In addition, for many combustion processes, the production of soot is an indicator of incomplete combustion and therefore a loss in chemical energy utilization.

The fundamental challenge in predicting soot formation lies in the fact that soot formation is heterogeneous formation process. Recent studies have shown that the early polycyclic aromatic hydrocarbon (PAH) substructures, that lead up and create incipient soot particles, need to account for the early time morphology behavior. ${ }^{3,4}$ Many existing descriptions of soot formation processes do not account for the morphology dependent nucleation processes which may explain, in part, the reason for current discrepancies between the observed and predicted amounts of soot formed for even well controlled systems. ${ }^{5,6}$

Recently Violi et al. $^{7,8}$ have developed a unique multiscale approach to characterize particle formation, through the use of atomistic models, such as kinetic Monte Carlo technique and molecular dynamics. This code, named atomistic model for particle inception (AMPI), makes it possible to follow the transformations that occur during particle formation in a chemically specific way, providing information on both the chemical structure and the configuration of the nanoparticles. The main thrust of this approach is to provide the missing microscopic detail of the molecular growth, which is experimentally inaccessible, using large-scale, statistical mechanics simulations and molecular dynamics.

In this paper, the AMPI code's methodology is presented

\footnotetext{
${ }^{\text {a)}}$ Electronic mail: avioli@umich.edu

${ }^{b}$ Present address: Department of Chemistry, University of Utah, Salt Lake City, UT 84112.
}

in detail and it is applied to study the formation of carbonaceous nanoparticles in an aromatic premixed flame. The aromatic growth process strongly depends on the specific local regime, which is characterized by several experimental factors including temperature, concentration of hydrogen, $\mathrm{PAH}$, etc., and it is, therefore, interesting to assess the influence of different environments on structural properties of the compounds formed.

The strength of this approach is that it provides information on the physical as well as the chemical structure of soot precursors. In this paper we compute for the first time density, porosity, and other physical properties of the nanoparticles as they evolve in time.

After summarizing in the following section the details of the statistical and deterministic methodologies, a thorough description of the synergistic combination of kinetic Monte Carlo and molecular dynamics methods into the AMPI code is reported, and the results of the molecular growth process are discussed in a premixed laminar benzene flame.

\section{METHODOLOGY}

Particle growth occurs on a time scale that is generally not accessible to fully atomistic approaches such as molecular dynamics, whereas an atomically resolved Monte Carlo method parametrized by accurate chemical kinetic data is capable of exploring growth over long times and large length scales. In order to reach long time scales while retaining the full atomistic detail, the kinetic Monte Carlo algorithm has been coupled to the molecular dynamics (MD) module, generating the AMPI code. ${ }^{9,10}$

Below we first describe the characteristics of the individual modules (kinetic Monte Carlo and molecular dynamics) and then their coupling within the AMPI code. 


\section{Molecular dynamics}

Our focus is on simulating the dynamical evolution of systems of atoms and MD represents the best tool for this class of problems. MD implements numerical solutions of Newton's equations of motion on an atomistic model of a molecular system to obtain information about its timedependent properties. This requires first choosing an interatomic potential for the atoms and then a set of boundary conditions. Integrating the classical equations of motion forward in time, the behavior of the system emerges naturally, requiring no intuition or further input from the user.

A serious limitation, however, is that accurate integration requires time steps short enough $\left(\sim 10^{-15} \mathrm{~s}\right)$ to resolve the atomic vibrations. Consequently, the total simulation time is typically limited to less than $1 \mu \mathrm{s}$, while the particle formation processes we wish to study take place on much longer time scales. This is the "time-scale problem."

\section{Kinetic Monte Carlo}

Kinetic Monte Carlo ${ }^{11-16}$ attempts to overcome this limitation by exploiting the fact that the long-time dynamics of this kind of system typically consist of jumps from state to state. Rather than following the trajectory through every vibrational period, these state-to-state transitions are treated directly. The result is that kinetic Monte Carlo (KMC) can reach vastly longer time scales, typically seconds and often well beyond.

Let us assume that our system is currently in state $i$, and we have a set of pathways, $M$, and associated rate constants $k_{i j}$. For each of these pathways, the probability distribution for the first escape time is

$$
p_{i j}(t)=k_{i j} \exp \left(-k_{i j} t\right)
$$

The pathway selection procedure proceeds as follows. We imagine that for each of the $M$ escape pathways we have an object with a length equal to the rate constant $k_{i j}$ for that pathway. The sum of these objects gives a total length $k_{\text {tot }}$. Let array element $s(j)$ represent the length of all the objects up to and including object $j$,

$$
s(j)=\sum_{q}^{j} k_{i q} .
$$

A random number $r$, distributed on $(0,1)$, is then drawn and it is multiplied by $k_{\text {tot }}$. Stepping through the array $s$, the module will stop at the first element for which $s(j)>r k_{\text {tot }}$. This is the selected pathway. To advance the clock, we draw a random time from the exponential distribution for the rate constant $k_{\text {tot }}$.

$$
\delta t_{\mathrm{KMC}}=\frac{-\ln (r)}{k_{\mathrm{tot}}} .
$$

The time advance has nothing to do with which event is chosen. The time to escape depends only on the total escape rate. Once the system is in the new state, the list of pathways and rates is updated and the procedure is repeated.
This procedure is often referred as the BKL algorithm (or the $n$-fold way algorithm), due to the 1975 paper by Bortz, Kalos, and Lebowitz. ${ }^{11}$

\section{AMPI: A synergistic combination of $\mathrm{KMC}$ and MD}

The AMPI code combines the strengths of the two methodologies in a unique way. MD can be very useful for exploration of the local phase space whereas the KMC method may be more effective for conformational changes which jump to a completely different area of phase space. This feature represents the peculiarity of the new proposed methodology; the two approaches have been widely used ${ }^{17}$ but never been integrated in this way. The code places the two simulation procedures on an equal footing and involves alternating between MD and KMC steps during the simulation.

The KMC algorithm is used to explore the most probable reaction events during carbonaceous nanoparticle growth and formation; MD simulations are employed to relax the growing particle in between reaction events thereby exploring the local phase space.

The combination of the two techniques spans two time scales: MD allows for relaxation, while the KMC part allows much larger time-scale changes to the system, provided that the system is at equilibrium. The time step for a single KMC iteration is a "real time," determined by the kinetics system. The result is a rapidly convergent method, which is able to solve the sampling problem with many organic molecules and complexes. The reaction rates among the compounds present in the system are specified as probabilities and the surface configuration over time is then given by a master equation, describing the time evolution of the probability distribution of system configurations.

\section{Inputs to the AMPI code}

The AMPI code consists of three modules: (a) gas-phase species, (b) reaction sites, and (c) reaction rates. For the gasphase input, the user defines the temperature, and the number and concentrations of the gas-phase species that contribute to the formation of the nanoparticles. Some examples of common gas-phase species are $\mathrm{H}, \mathrm{H}_{2}$, aliphatic, and aromatic species, such as $\mathrm{CH}_{3}, \mathrm{C}_{6} \mathrm{H}_{6}, \mathrm{C}_{10} \mathrm{H}_{8}$, and their corresponding radicals.

Once the species are defined, it is important to list the possible reactions that can occur over time and describe them in terms of reaction sites, i.e., atoms where a particular reaction can occur. The reaction site module governs the definition and counting algorithm of reaction sites, which are capable of undergoing modification (for example, addition reactions). Some examples of reaction sites are (a) atom on a five or six membered ring, (b) $s p^{3}$ carbon, (c) HCCCCR and HCCCCCR chains prone to close to form five and six membered rings where $R$ is a radical carbon, and (d) $\mathrm{HCCCCCH}$ and $\mathrm{HCCCCCCH}$ to form five and six membered rings via the route of dehydrogenation.

Proximity conditions are also placed on radical sites. In the site-counting procedure, a radical carbon is considered as 
a possible site for termination or addition by a gas-phase species only if the gas-phase species of interest can be accommodated.

The third input to the AMPI code is the list of the rate constants. These data have been obtained either from literature or $a b$ initio calculations. The reactions included in the AMPI code can be broadly classified into three categories.

\section{Ring closure reactions}

Ring closure reactions involve the formation of nascent rings through dehydrogenation mechanisms. Two hydrogen atoms are lost, and a new ring is created by the formation of a new transannular $\mathrm{C}-\mathrm{C}$ bond. Recently results relative to new reaction pathways for the cyclodehydrogenation of PAH to form cyclopentafused aromatic hydrocarbons have been reported by $\mathrm{Violi}^{18}$ and the computed reaction rates have been included in this version of the code.

\section{Abstraction reactions}

An example of abstraction reaction can be expressed as $R_{i}-\mathrm{H}+\mathrm{H}=R_{i}+\mathrm{H}_{2}$, where $R_{i}-\mathrm{H}$ is a saturated site on the growing structure and $R_{i}$ is its radical. Usually this initiation step is represented by $\mathrm{H}$ abstraction reactions, which activate the aromatic structures. This can be accomplished in many different ways, but at conditions of typical flame and shocktube experiments $\mathrm{H}$ abstraction by a gaseous $\mathrm{H}$ typically dominates. $^{19-21}$

\section{Addition reactions}

Addition reactions are divided into two categories: termination and elimination reactions. In a termination reaction, a reactive intermediate is rendered inactive, thus ending the reaction sequence.

In an elimination reaction, two groups are removed from a molecule in either a one- or two-step mechanism. Either the unsaturation of the compound increases or the valence of an atom in the molecule decreases by 2 (this is known as reductive elimination).

$$
R_{i}+R_{j}-\mathrm{H}=R_{i}-R_{j}+\mathrm{H} .
$$

Here, the radical $R_{i}$ is added to the neutral site $R_{j}-\mathrm{H}$ on the growing structure, removing a hydrogen atom. ${ }^{22-24}$

For these type of reactions, the sites identified by the code are $\mathrm{HCCCCC}^{*} / \mathrm{HCCCCCC}^{*}$ chains that are prone to close to form five/six membered rings, and $\mathrm{HCCCCCH} / \mathrm{HCCCCCH}$ chains that are prone to close to form five membered rings. In a situation such as the one defined by the first set of sites, the code removes the terminal $\mathrm{H}$ from the chain and then closes the ring. With the second set of sites, the code removes the two terminal hydrogens and forms a new ring. 10,25

MD simulations allow for relaxation of the molecules towards thermal equilibrium. Within the MD module, the potential used to describe hydrocarbon interactions is the adaptive intermolecular reactive bond order (AIREBO), ${ }^{26}$ based on the reactive empirical bond-order potential of Brenner et al. ${ }^{27,28}$ The potential describes the covalent bonding interactions largely as in the REBO potential of Brenner et $a{ }^{29}$ In addition, there are terms in the energy to account for torsional energies of single-bond rotations, as well as the nonbonded van der Waals interactions. The torsional energy is modeled as a pairwise interaction between atoms. The advantage of this formulation is that the symmetry of the dihedral angle potential is dictated by the symmetry of the molecule and can change as reactions occur. The van der Waals interaction is modeled using the Lennard-Jones 12-6 potential. The thermodynamic ensemble realized for the MD module is the $(N, V, T)$ canonical ensemble, where the number of particles, the volume, and the temperature are fixed to prescribed values. The temperature is the intensive parameter. Different methods were proposed to fix the temperature to a fixed value during the simulation without allowing fluctuations of temperature. The weak collision (WC) model is used here. For each MD time step, the WC model randomly reassigns velocities of a portion of the atoms based on the Boltzmann distribution at a given temperature. The predictor corrector method $^{30}$ was used to integrate the equations of motion.

Bonding pairs in the reaction sites described above are defined according to the formalism of Brenner et al. Hence, chains and rings are identified through efficient nested loops directed by neighbor list pointers. Additional proximity and geometric conditions are placed on the definition of the $\mathrm{HC} \cdot \mathrm{CH}$ chains to ensure that false-positive sites are not identified.

\section{AMPI algorithm}

The algorithm used by the AMPI code is described as follows.

(1) The code reads a set of inputs, such as temperature, number, and concentrations of species in the gas phase, reaction rates, and MD related quantities.

(2) It counts the number of sites available for reactions on the growing structure by identifying each atom as radical, neutral, aliphatic, and aromatic sites. Then the code checks for steric hindrance, and the reactions available at that time are classified as either accepted or rejected.

(3) The code computes the kinetic rate constants for the accepted reactions $\left(k=A T^{n} \exp \left(-E_{a} / R T\right)\right)$ and their corresponding reaction rates.

(4) Once a list of possible reactions is created along with possible sites and reaction rates, the code randomly selects a reaction based on the reaction probability that is proportional to its reaction rate.

(5) It randomly selects the site for the reaction to occur, assuming that all possible sites for this reaction have an equal probability.

(6) It then modifies the structure of the growing particle according to the selected reaction: if abstraction is selected, remove the selected atom(s) from the sites from which the species should be abstracted. If addition is chosen, add the fragment to the selected site; for ring closure, identify the atoms which need to form a bond to obtain a ring and the atoms which undergo dehydrogenation before the ring closure. 
(7) The clock is then incremented in a way that is consistent with the average time for escape from that state, which can be determined easily from the rate constants for the possible escape paths in the list.

(8) Finally, the code relaxes the newly formed structure using a MD run. MD allows for relaxation of the molecules towards thermal equilibrium.

\section{RESULTS}

A low-pressure benzene-oxygen-argon flame (equivalence ratio $\Phi=1.8$ ) with an unburned velocity of $50 \mathrm{~cm} \mathrm{~s}^{-1}$, $P=2.63 \mathrm{kPa}$ is used in this paper as environment within which particles are grown. This flame has been experimentally studied by Bittner and Howard, ${ }^{31}$ who measured the concentration profiles of radicals and stable species using a molecular beam/mass spectrometer. ${ }^{32}$

Bittner and Howard reported the signal from species larger than 200 and $700 \mathrm{u}$ (unified atomic mass unit). They did not calibrate their spectrometric signal, but the shape of the curve gives information on the particle inception region. In particular, the curve for $200 \mathrm{u}$ starts at a distance of $0.5 \mathrm{~cm}$ from the burner, it rapidly increases and maximizes at about $0.8 \mathrm{~cm}$. The species concentration profile then decreases across the end of the main oxidation zone. Drawing on these data, two locations $(x=0.59$ and $0.78 \mathrm{~cm})$ were chosen in this study to study the formation of carbon nanoparticles, corresponding to temperatures of $1450 \mathrm{~K}$ at $0.59 \mathrm{~cm}$ and $1765 \mathrm{~K}$ at $0.78 \mathrm{~mm}$.

The experimental data in terms of temperature and concentration profiles of the gas-phase species are supplied as inputs to the code. The current AMPI code includes $\mathrm{H}, \mathrm{H}_{2}$, $\mathrm{CH}_{4}, \mathrm{CH}_{3}, \mathrm{C}_{2} \mathrm{H}_{2}, \mathrm{C}_{2} \mathrm{H}, \mathrm{C}_{2} \mathrm{H}_{3}, \mathrm{C}_{2} \mathrm{H}_{5}, \mathrm{C}_{3} \mathrm{H}_{3}, \mathrm{C}_{4} \mathrm{H}_{3}, \mathrm{C}_{4} \mathrm{H}_{5}$, $\mathrm{C}_{5} \mathrm{H}_{5}, \mathrm{C}_{5} \mathrm{H}_{6}$, acenaphthylene, naphthalene, biphenyl, phenylacetylene, styrene, and their corresponding radicals, as reacting gas-phase species.

The gas-phase concentrations of the main species used in the AMPI code together with the temperatures are reported in Table I.

For each point in the flame, a statistical ensemble of nanoparticles was generated using the AMPI code with different initial seeds, e.g., benzene, naphthalene, etc., and various random numbers.

The total simulation time was obtained as the sum of the KMC and elapsed MD times. Using the AMPI algorithm described earlier, for each KMC move, i.e., reaction event, a MD run was performed until the particle was equilibrated. The convergence criterion for the equilibration phase is based on the average temperature per atom approaching the set point temperature.

After equilibration of the particle, the MD run was continued for an additional $10 \mathrm{ps}$ to ensure adequate relaxation. The instantaneous structures (geometries) from the last time step of the MD module are used for the next KMC reaction.

The concentration of gas-phase precursors differ at various points in the flame and this strongly influences the formation of carbon nanoparticles at these locations. The relative contributions of the type of reactions change as combustion progresses, with compounds such as benzene
TABLE I. Concentration (in mole fraction) of gas-phase species present in the inception region.

\begin{tabular}{lcc}
\hline \hline Gas-phase species & $\begin{array}{c}x=0.59 \mathrm{~cm} \\
(T=1410 \mathrm{~K})\end{array}$ & $\begin{array}{c}x=0.78 \mathrm{~cm} \\
(T=1765 \mathrm{~K})\end{array}$ \\
\hline $\mathrm{C}_{5} \mathrm{H}_{5}$ & $7.04 E-5$ & $1.05 E-3$ \\
$\mathrm{C}_{5} \mathrm{H}_{6}$ & $1.71 E-3$ & $2.85 E-4$ \\
$\mathrm{C}_{6} \mathrm{H}_{5}$ & $5.00 E-6$ & $1.37 E-4$ \\
$\mathrm{C}_{6} \mathrm{H}_{6}$ & $7.50 E-2$ & $2.00 E-2$ \\
Toluene & $8.00 E-5$ & $2.00 E-4$ \\
$\mathrm{Xylene}_{2}$ & $2.64 E-8$ & $3.68 E-9$ \\
$\mathrm{C}_{6} \mathrm{H}_{6} \mathrm{C}_{2} \mathrm{H}$ & $2.50 E-4$ & $3.89 E-4$ \\
$\mathrm{C}_{6} \mathrm{H}_{5} \mathrm{C}_{2} \mathrm{H}_{2}$ & $6.08 E-6$ & $2.73 E-6$ \\
$\mathrm{C}_{6} \mathrm{H}_{5} \mathrm{C}_{2} \mathrm{H}_{3}$ & $6.08 E-5$ & $3.31 E-5$ \\
Indenyl & $5.15 E-6$ & $2.19 E-5$ \\
1-naphthyl & $0.15 E-6$ & $2.53 E-6$ \\
2-naphthyl & $0.15 E-6$ & $3.32 E-6$ \\
Naphthalene & $1.71 E-4$ & $2.26 E-4$ \\
Bipheny radical & $4.04 E-6$ & $4.79 E-6$ \\
Biphenyl & $4.04 E-5$ & $4.79 E-5$ \\
4-acenaphthyl & 0 & $3.79 E-7$ \\
Acenaphthalene & $3.45 E-5$ & $1.23 E-4$ \\
\hline
\end{tabular}

and cyclopentadiene dominating early in the flame and acetylene, propargyl, $\mathrm{C}_{4} \mathrm{H}_{2}, \mathrm{C}_{5} \mathrm{H}_{5}$ derivatives dominating at later stages of combustion. An allowance is made for the change in the gas-phase concentrations at different heights in calculating the soot precursor composition, but the depletion in gas-phase species by the formation of precursors is neglected.

Table II reports the list of the reactions that significantly contribute to the nanoparticles growth at $x=0.59$ and $x$ $=0.78 \mathrm{~cm}$. A list of the reaction rates used for these calculations is provided as supporting information.

The percentage of reactions undergoing ring closure (de-

TABLE II. Some key reactions in the particle inception zone of benzene flame.

\begin{tabular}{lcc}
\hline \hline & $\begin{array}{c}\text { Percentage } \\
\text { at } x=0.59 \mathrm{~cm} \\
T=1450 \mathrm{~K}\end{array}$ & $\begin{array}{c}\text { Percentage at } \\
x=0.78 \mathrm{~cm} \\
T=1765 \mathrm{~K}\end{array}$ \\
Reaction & 18 & 26 \\
Cyclodehydrogenation reactions & & \\
& & \\
Abstraction reactions & 2.9 & 0.8 \\
$\mathrm{CH}_{3}$ radical & 13.0 & 22.0 \\
$\mathrm{C}_{2} \mathrm{H}$ radical & 4.2 & 2.7 \\
$\mathrm{C}_{5} \mathrm{H}_{5}$ radical & 2.5 & 4.3 \\
$\mathrm{C}_{6} \mathrm{H}_{5}$ radical & & \\
$\mathrm{Addition}$ reactions & 2.1 & \\
$\mathrm{C}_{6} \mathrm{H}_{5} \mathrm{C}_{2} \mathrm{H}, \mathrm{C}_{6} \mathrm{H}_{5} \mathrm{C}_{2} \mathrm{H}_{2}$ & 0.8 & 0.8 \\
$\mathrm{C}_{3} \mathrm{H}_{3}$ to five/six rings & 0.4 & 2.3 \\
$\mathrm{C}_{5} \mathrm{H}_{5} / \mathrm{C}_{6} \mathrm{H}_{5}$ to five/six rings & 7.1 & 2.3 \\
$\mathrm{CH}_{3}$ to five/six rings & 0.4 & 2.7 \\
$\mathrm{C}_{2} \mathrm{H}_{2}$ to five/six & 12.2 & 0.8 \\
$\mathrm{C}_{2} \mathrm{H}_{4}$ to aliphatic carbons & 7.1 & 6.6 \\
$\mathrm{C}_{3} \mathrm{H}_{3}$ to five/six rings & 0.4 & 2.3 \\
$i-\mathrm{C}_{4} \mathrm{H}_{3}$ to five/six rings & 11.0 & 0.8 \\
$i-\mathrm{C}_{4} \mathrm{H}_{3}$ to nonaromatic $\mathrm{C}$ & 8.8 & 15.1 \\
$\mathrm{C}_{5} \mathrm{H}_{5} / \mathrm{C}_{6} \mathrm{H}_{5}$ to five/six rings & & 8.5 \\
\hline
\end{tabular}




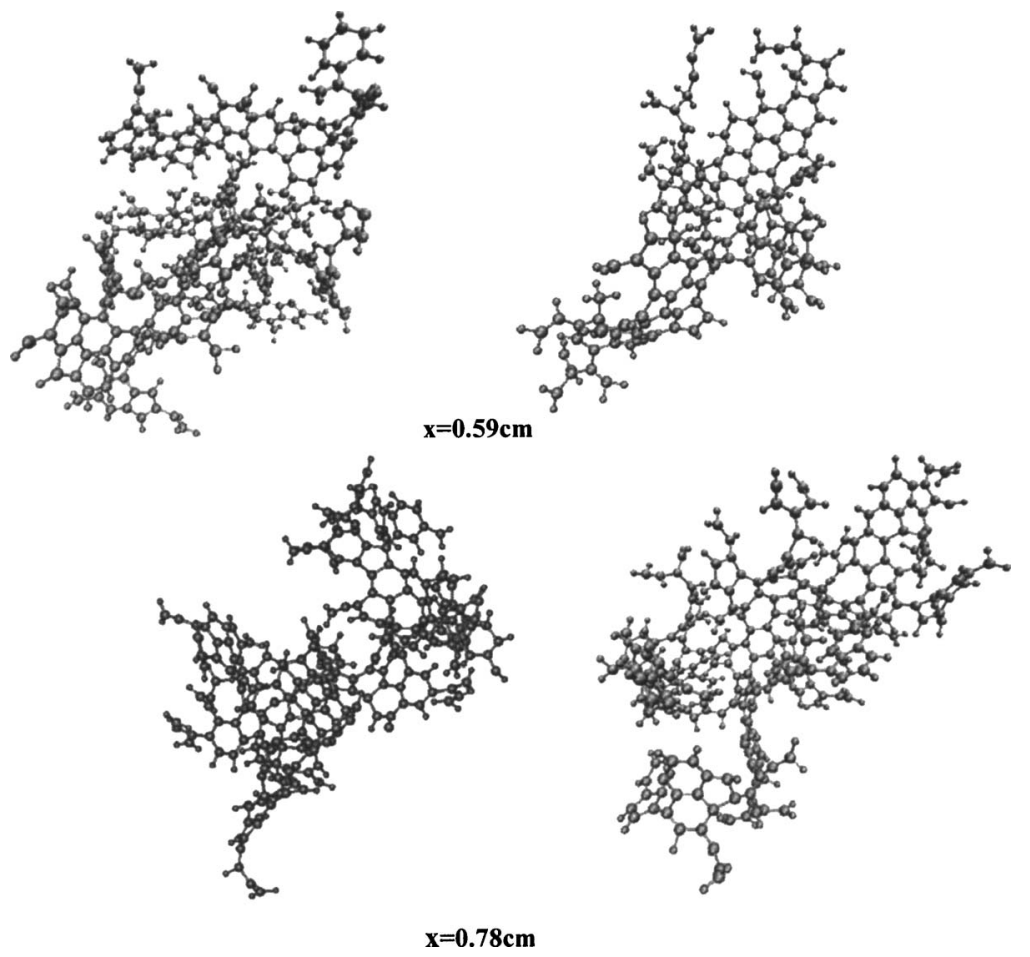

FIG. 1. Examples of carbonaceous nanoparticles produced by the AMPI code in a premixed benzene flame at $x=0.59 \mathrm{~cm}$ (top panel) and $0.78 \mathrm{~cm}$ (lower panel). Carbon atoms are shown in blue and hydrogen atoms are displayed in silver. hydrogenation where either there is a loss of one hydrogen atom or both hydrogen atoms followed by the formation of a new ring) is approximately $18 \%$ and $24 \%$ at 0.59 and $0.78 \mathrm{~cm}$, respectively, as reported in Table II.

Within these two zones, the temperature rises and the number of vibrational degrees of freedom of the PAH increases with growth. These factors favor unimolecular reactions of the PAH that normally have larger activation energies than addition reactions with unsaturated hydrocarbons. Unimolecular reactions of large PAH lead to structures that are more reactive to addition of smaller unsaturated hydrocarbons. $^{33}$

There is a competition between bimolecular association reactions (addition or growth reactions) and rearrangement reactions, which exhibit higher activation energies: at the low temperatures in the beginning of the oxidation zone, the molecules grow but rearrangement and degradation reactions do not play a significant role. With increasing temperature the growth reactions accelerate but the rates of rearrangements also increase. Cage closure reactions, breaking $\mathrm{C}-\mathrm{H}$ bonds and intramolecular rearrangements, have large activation energies and are favored at higher temperature.

An important observation is the ratio of hydrogen addition to hydrogen abstraction. This ratio is lower at $x$ $=0.78 \mathrm{~cm}$ than at $0.59 \mathrm{~cm}$ and hence the termination reactions are more likely to happen at $x=0.78$. At $x=0.59$ and $0.78 \mathrm{~cm}$, the addition of $\mathrm{CH}_{3}$ to neutral sites on five/six membered ring, $\mathrm{C}_{2} \mathrm{H}_{4}$ addition to radical sites on aliphatic carbons, $i-\mathrm{C}_{4} \mathrm{H}_{3}$ addition to neutral sites on nonaromatic carbons, and $\mathrm{C}_{5} \mathrm{H}_{5} / \mathrm{C}_{6} \mathrm{H}_{5}$ addition to neutral sites on five/six member rings play key roles in the particle growth. The most recurrent abstraction reactions involve $\mathrm{CH}_{3}, \mathrm{C}_{2} \mathrm{H}, \mathrm{C}_{5} \mathrm{H}_{5}$, and phenyl abstractions. Among them, $\mathrm{C}_{2} \mathrm{H}$ abstraction happens as frequently as the addition of $i-\mathrm{C}_{4} \mathrm{H}_{3}$ to neutral sites on nonaromatic carbons. Thus, there is a strong competition between abstraction and addition reactions during the growth of these carbon nanoparticles.

Figure 1 shows some typical structures formed in flame. One of the features of these nanoparticles is the considerable presence of five and six membered rings. On average, at $x$ $=0.59 \mathrm{~cm}$, nanoparticles are composed for the $12 \%$ of five member rings and $15.1 \%$ of six member rings. The values decrease to $10.1 \%$ and $16.7 \%$, respectively at $x=0.78 \mathrm{~cm}$.

\section{Aspect ratio}

One of the important characteristics of these particles is that their morphology and ellipticity parameters are often used to characterize particle shape. ${ }^{34}$ The aspect ratio (AR) is computed as

$$
\mathrm{AR}=a / b,
$$

where $a$ and $b$ are the axes of the Legendre ellipse that has the same geometrical moments up to the second order as the original object area.

A series of diameters of the particles is sampled. The average of these diameters is used as the spherical diameter $r_{s}$ of the configurations, and an average of the diameters longer than $r_{s}$ is used as $a$, and an average of the shortest ones is used as $b$.

The aspect ratio of the particle, defined as $a / b$, is reported in Figs. 2(a) and 2(b) as a function of time for the two locations in the flame $(x=0.59 \mathrm{~cm}$ and $x=0.78 \mathrm{~cm})$. The data are relative to 20 averaged simulation runs for each point in the flame. The AR of a circle is 1 and of an ellipse with the ratio of axes $2: 1$ is equal to 2 . The computational results obtained with the AMPI code shows an increase in the aspect ratio with increasing particle size, reaching the value if 1.2.

The elongated shapes exhibited by the young nanopar- 

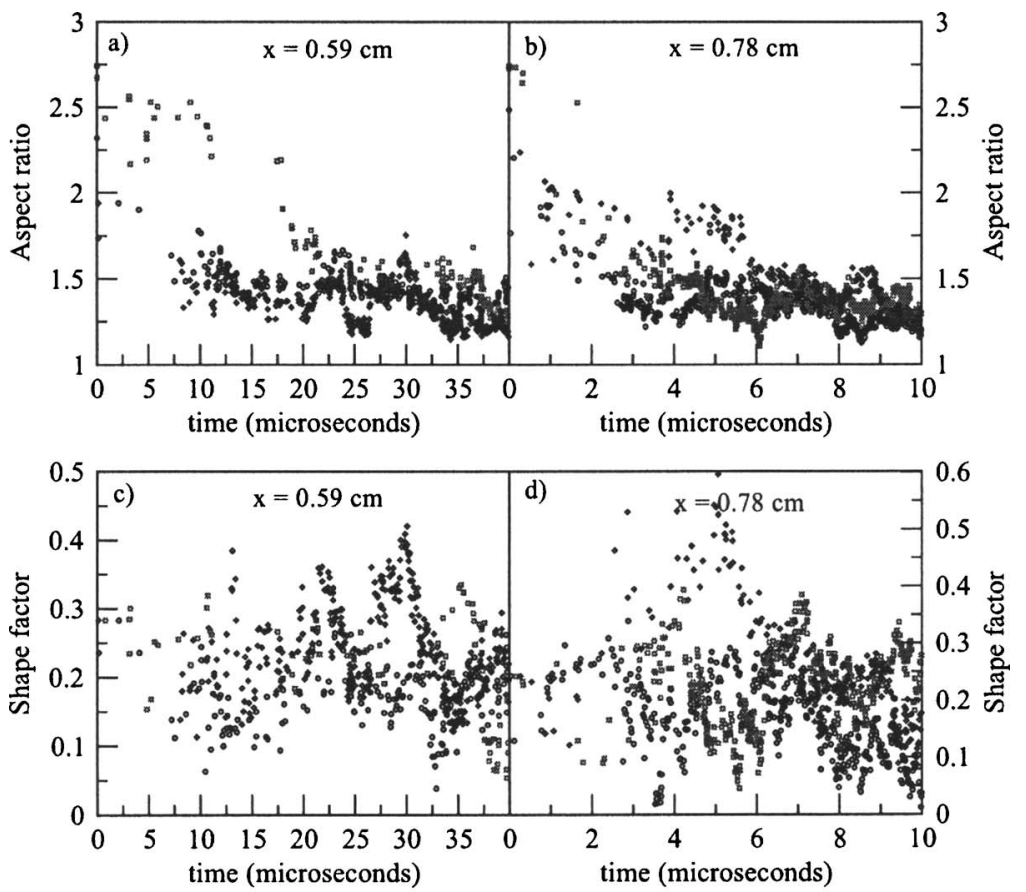

FIG. 2. Aspect ratio, shape factor, and diameter (nm) of growing carbonaceous nanoparticles at $x=0.59 \mathrm{~cm}[(\mathrm{a})$, (c), and (e) $]$ and $0.78 \mathrm{~cm}[(\mathrm{~b}),(\mathrm{d})$, and (f) $]$ as function of time for three different simulations.

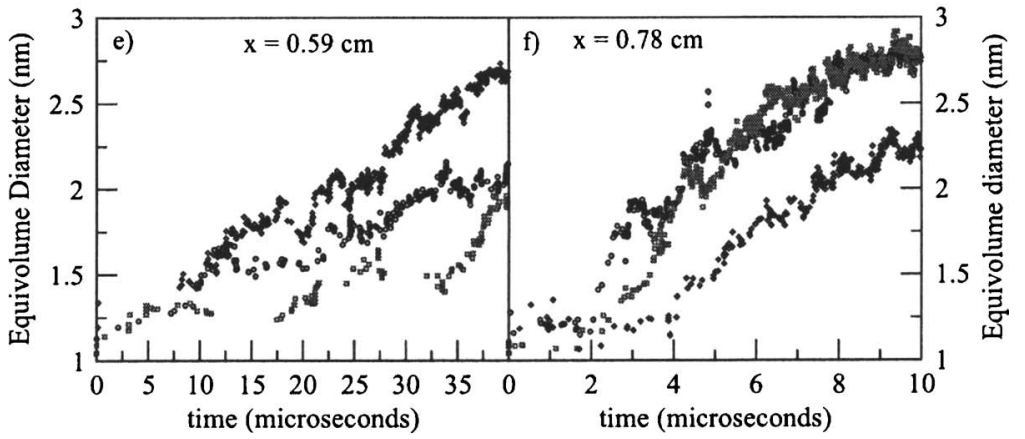

ticles are supported by in situ measurements. Light scattered by nanoparticles is more depolarized than that scattered by soot particles and the depolarization ratio increases as the particles deviate from the spherical shape ${ }^{35}$ The spherical deviation is further supported by an examination of the shape factor $\left(S_{f}\right)$ (Ref. 36) of these nanoparticles at both points in the flame, as shown in Figs. 2(c) and 2(d).

$$
S_{f}=r_{\text {rmsd }} / r_{\text {mean }}
$$

where $r_{\text {mean }}$ is the mean radius length and $r_{\text {rmsd }}$ is the root mean square deviation of radius lengths defined as

$$
r_{\text {rmsd }}=\sqrt{\frac{\left(r_{\text {max }}-r_{\text {mean }}\right)^{2}+\left(r_{\text {max }}-r_{\text {mean }}\right)^{2}}{2}} .
$$

Here, $r_{\min }$ and $r_{\max }$ are the minimum and maximum radius lengths. For example, the shape factor for a perfect circle or sphere is 0 , whereas for a star shaped object it is 0.36 . In other words, lower the shape factor of an object, closer it tends towards a sphere. The diameters of many of these nanoparticles are reported in Figs. 2(e) and 2(f) and they vary from 1.75 to $2.25 \mathrm{~nm}$.

The growth rates are very different at the two locations. At lower temperatures, it takes on average of $40 \mu$ s to produce a particle of $2.5 \mathrm{~nm}$ in diameter, while at $x=0.78 \mathrm{~cm}$
$10 \mu$ s are sufficient to generate structures of $2.7 \mathrm{~nm}$ in size. This is due to the high occurrence of termination and addition reactions at lower temperature.

\section{Density}

The mass density $\left(D_{p}\right)$ of each particle produced by the AMPI code is computed as

$$
D_{p}=M_{n p} / V_{m}
$$

where $M_{n p}$ is the total mass of the particle and $V_{m}$ is the molecular volume computed using Connolly's algorithm. ${ }^{37}$ The average mass density is then obtained from the individual mass densities of each particle. The average densities of these nanoparticles at $x=0.59$ and $0.78 \mathrm{~cm}$ are $1.44 \mathrm{~g} / \mathrm{cm}^{3}$ [Fig. 3(a)] and $1.5 \mathrm{~g} / \mathrm{cm}^{3}$ [Fig. 3(b)]. These values are close to the density of bituminous coal $\left(1.48 \mathrm{~g} / \mathrm{cm}^{3}\right)$ as reported by Faulon et $a l^{38}$

\section{Porosity}

In order to compute the porosity of a particle, the pore volume is determined based on the choice of specific probe radii. Particle porosity influences the diffusion of gas-phase species and surface reactions. Porosity $(P)$ is defined as the ratio between the volume of the pores in the particle and the probe radius. The choice of probe radii is dependent on the 


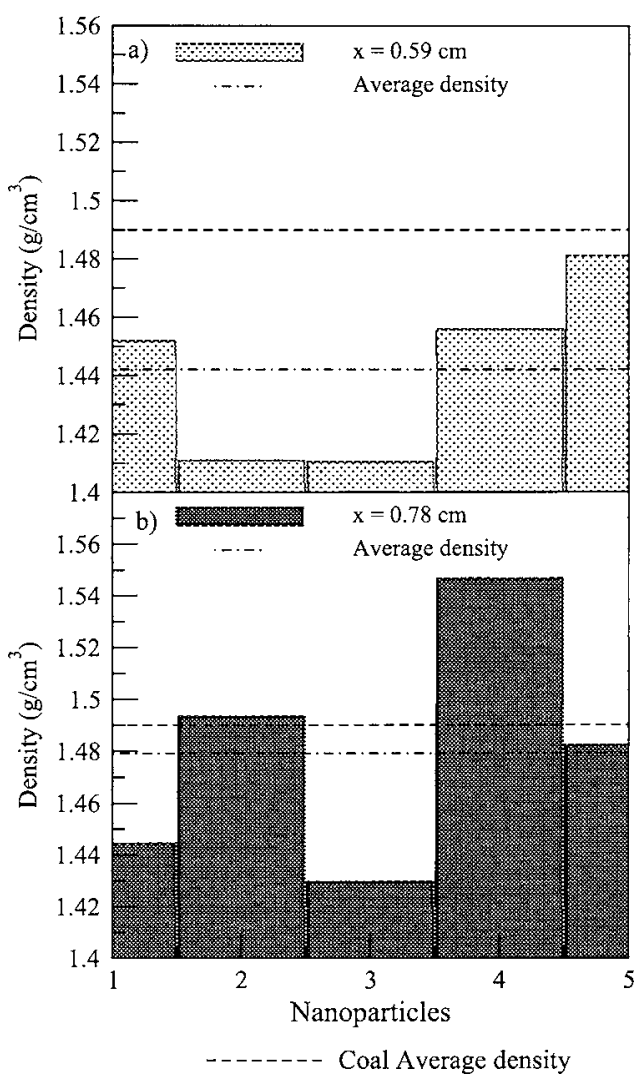

FIG. 3. Density histograms of carbonaceous nanoparticles at $x=0.59 \mathrm{~cm}$ (a) and $0.78 \mathrm{~cm} \mathrm{(b).}$

nature of diffusing molecules and it affects the calculation of the pore volume. As an example, the probe radius for diffusion of helium through the carbon nanopores is $0.254 \mathrm{~nm}$, whereas for $\mathrm{N}_{2}$ it is $0.286 \mathrm{~nm}$ as calculated by Rao. ${ }^{39}$ Similar to the mass density, the average porosity is computed from the porosity of each particle for fixed probe radii.

The porosity of carbonaceous particles is obtained using the choice of probe radius equal to $0.286 \mathrm{~nm}$ since it is the critical distance for diffusion of nitrogen gas through these nanopores [Figs. 4(a) and 4(b)]. The average porosity at $x$ $=0.59 \mathrm{~cm}$ is 0.37 whereas at $x=0.78 \mathrm{~cm}$ it is 0.46 . Higher the porosity of the particle, greater is the probability of diffusion of gases which are present in the flame.

\section{Radial distribution functions}

Radial distribution functions ${ }^{31}$ (RDFs) are typically computed to describe qualitatively the behavior of the structures. The RDF $g(r)$ can be expressed as

$$
g(r)=\frac{\operatorname{nhist}(r)}{N_{a} \tau 4 \pi r^{2} d r},
$$

where nhist $(r)$ is the number of bins in the histogram, which falls between $r$ and $r+d r, N_{a}$ is the number of atoms in any particle, and $\tau$ is the number of trajectories for which the histograms are stored.

The RDFs obtained from an ensemble of nanoparticles at $x=0.59$ and $0.78 \mathrm{~cm}$ are shown in Figs. 5(a) and 5(b). Since the amplitude and characteristics of the aromatic RDF curves

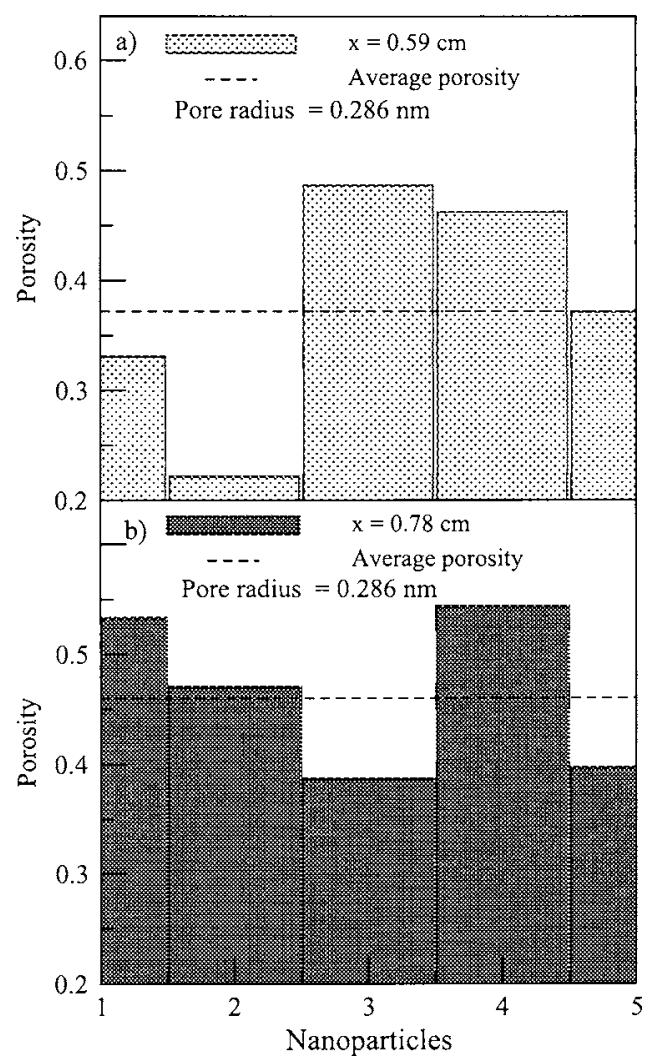

FIG. 4. Porosity histograms of carbonaceous nanoparticles for probe radius of $0.286 \mathrm{~nm}$ at $x=0.59 \mathrm{~cm}$ (a) and $0.78 \mathrm{~cm} \mathrm{(b).}$

are similar to the total RDF curves in both cases, the nanoparticles produced at $x=0.59$ and $x=0.78 \mathrm{~cm}$ are predominantly aromatic in nature.

In both cases, the first peak of the aromatic RDF curves appears at $r=1.41 \AA$, which is the carbon-carbon bond distance in benzenelike structures. At $x=0.78 \mathrm{~cm}$, the second peak is around $2.47 \AA$ (similar to the carbon-carbon second nearest neighbors in benzene) while at $x=0.59 \mathrm{~cm}$ the peak is shifted towards larger distances. A similar observation can be derived for the third peak. If the particles contain mainly benzenelike structures the peak should appear at $r=2.85 \AA$. The aliphatic RDF curves significantly contribute to the total RDFs between 8 and $18 \AA$. The probability of finding any aliphatic fragments below $8 \AA$ is low, suggesting the presence of mainly PAH below $8 \AA$.

These observations are consistent with a fast formation of PAH in the main flame region, which react among themselves, growing in size and then they are rapidly consumed at the flame front by the pyrolysis and oxidative pyrolysis of the high molecular mass species. In the $\mathrm{C}_{6} \mathrm{H}_{6}$ flame, the concentration of particles peaks before the end of the main oxidation zone, then drops across the maximum temperature zone, and levels off with respect to the distance from the burner.

\section{CONCLUSIONS}

A multiscale atomistic code, AMPI, has been applied to study the chemical and physical properties of carbonaceous nanoparticles formed in a premixed laminar benzene flame. The aspect ratio, size, diameter, density, and porosity were 


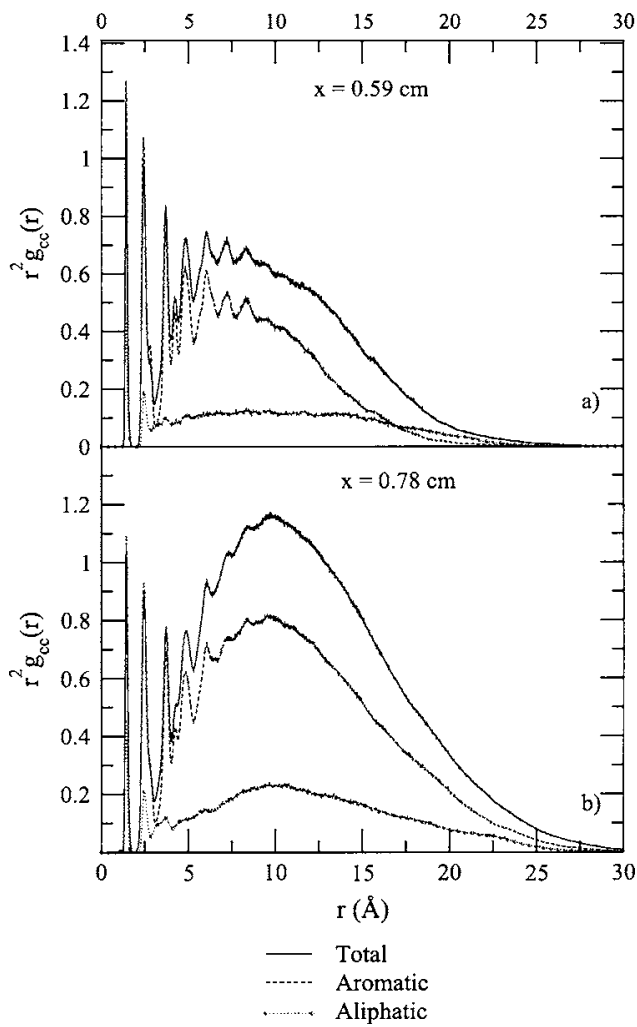

FIG. 5. Average carbon-carbon radial distribution functions for carbonaceous nanoparticles at $x=0.59 \mathrm{~cm}$ (a) and $0.78 \mathrm{~cm} \mathrm{(b).}$

computed from the atomistic morphology of the nanoparticles. Interesting results are reported in terms of shape and composition and how they change with the local environment of the flame. In the benzene flame the building blocks for larger aromatic formation are already present from the beginning of the oxidation process so that higher aromatics are massively formed in the main oxidation zone of the flame. The increase of temperature at the flame front reduces their concentration due to pyrolysis and/or oxidation resulting in a peak value in the main oxidation region and a decrease in the postoxidation zone of the flame.

The inclusion of more reactions into the AMPI code will help to better understand the relationship between reaction mechanisms and particle morphology and structural properties. Applications of the AMPI method to study carbonaceous nanoparticle formation in other aromatic and aliphatic fuel environments are ongoing.

\section{ACKNOWLEDGMENTS}

This research is funded by the National Science Foundation Grant No. CTS 0553764 and by the Nanoscale Interdisciplinary Research Team Grant No. EEC-0304433. The authors thank S. J. Stuart of Clemson University for providing the AIREBO force field. The authors also acknowledge the computer time support provided by the Center for High Performance Computing, University of Utah.

${ }^{1}$ G. Marland, T. A. Boden, and R. J. Andres, Global, Regional, and National Fossil Fuel CO2 Emissions. In Trends: A Compendium of Data on Global Change. Carbon Dioxide Information Center, Oak Ridge National Laboratory Report, U.S. Department of Energy, Oak Ridge, TN (2006).

${ }^{2}$ A. S. Manne, Stephan G. Energy Policy 27(6), 309-316 (1999).

${ }^{3}$ S. Izvekov and A. Violi, J. Phys. Chem. B 109, 17019 (2005).

${ }^{4}$ S. Izvekov and A. Violi, J. Chem. Theory Comput. 2, 504 (2006).

${ }^{5}$ M. Balthasar and M. Frenklach, Combust. Flame 140, 130 (2005).

${ }^{6}$ M. Balthasar and M. Frenklach, Combust. Flame 30, 1467 (2005).

${ }^{7}$ A. Violi, Combust. Flame 139, 279 (2004).

${ }^{8}$ A. Violi, G. A. Voth, and A. F. Sarofim, Proc. Combust. Inst. 30, 1343 (2005)

${ }^{9}$ A. Violi and G. A. Voth, High Performance Computing and Communications: Proceedings Lecture Notes in Computer Science 3726, 938-947 (2005).

${ }^{10}$ A. Violi, G. A. Voth, and A. F. Sarofim, Proc. Combust. Inst. 30, 1343 (2005).

${ }^{11}$ A. B. Bortz, M. H. Kalos, and J. L. Lebowitz, J. Comput. Phys. 17, 10 (1975).

${ }^{12}$ G. H. Gilmer, J. Cryst. Growth 36, 15 (1976).

${ }^{13}$ A. F. Voter, Phys. Rev. B 34, 6819 (1986).

${ }^{14}$ P. A. Maksym, Semicond. Sci. Technol. 3, 594 (1988).

${ }^{15}$ K. A. Fichthorn and W. H. Weinberg, J. Chem. Phys. 95, 1090 (1991).

${ }^{16}$ J. L. Blue, I. Beichl, and F. Sullivan, Phys. Rev. E 51, R867 (1995).

${ }^{17}$ J. B. McDermott, C. Libanati, C. La Marca, and M. T. Klein, Ind. Eng. Chem. Res. 29, 22 (1990).

${ }^{18}$ A. Violi, J. Phys. Chem. A 109, 7781 (2005).

${ }^{19}$ M. Frenklach, D. W. Clary, W. C. Gardiner, Jr., and S. E. Stein, Proc. Combust. Inst. 20, 887 (1985).

${ }^{20} \mathrm{H}$. Wang and M. Frenklach, Combust. Flame 110, 173 (1997).

${ }^{21}$ M. Frenklach and J. Warnatz, Combust. Sci. Technol. 51, 265 (1987).

${ }^{22}$ A. Violi, A. F. Sarofim, and T. N. Truong, Combust. Flame 126, 1506 (2001).

${ }^{23}$ A. D'Anna, A. Violi, and A. D'Alessio, Combust. Flame 121, 418 (2000).

${ }^{24}$ A. Violi, A. D'Anna, and A. D'Alessio, Chemosphere 42, 463 (2001).

${ }^{25}$ A. Violi, A. F. Sarofim, and G. A. Voth, Combust. Sci. Technol. 176, 991 (2004).

${ }^{26}$ S. J. Stuart, A. B. Tutein, and J. A. Harrison, J. Chem. Phys. 112, 6472 (2000).

${ }^{27}$ D. W. Brenner, Phys. Rev. B 42, 9458 (1990).

${ }^{28}$ D. W. Brenner, J. A. Harrison, C. T. White, and R. J. Colton, Thin Solid Films 206, 220 (1991)

${ }^{29}$ D. W. Brenner, O. A. Shenderova, J. A. Harrison, S. J. Stuart, B. Ni, and S. B. Sinnott, J. Phys.: Condens. Matter 14, 783 (2002).

${ }^{30}$ M. P. Allen and D. J. Tildesley, Computer Simulation of Liquids (Oxford University Press, New York, 1987).

${ }^{31}$ J. D. Bittner and J. B. Howard, Proc. Combust. Inst. 18, 1105 (1981).

${ }^{32}$ J. D. Bittner and J. B. Howard, in Particulate Carbon: Formation During Combustion, edited by D. C. Siegla and G. W. Smith (Plenum, New York, 1981), p. 109.

${ }^{33}$ A. Keller, R. Kovacs, and K.-H. Homann, Phys. Chem. Chem. Phys. 2, 1667 (2000).

${ }^{34}$ V. Mikli, H. Kaerdi, P. Kulu, and M. Besterci, Proc. Estonian Acad. Sci. Eng. 7, 22 (2001).

${ }^{35}$ A. D'Alessio, A. D'Anna, A. D'Orsi, P. Minutolo, R. Barbella, and A. Ciajolo, Proc. Combust. Inst. 24, 973 (1992).

${ }^{36}$ R. M. Carter and Y. Yuan, J. Phys.: Conf. Ser. 15, 177 (2005).

${ }^{37}$ M. L. Connolly, J. Am. Chem. Soc. 107, 1118 (1985).

${ }^{38}$ J.-L. Faulon, G. A. Carlson, and P. G. Hatcher, Energy Fuels 7, 1062 (1993).

${ }^{39}$ M. B. Rao, Carbon 29, 813 (1991). 